\title{
Supplementary Data for Manuscript:
}

\section{Matrix Library Screening of Substituted $N$-Aryl-1,8-Naphthalimides Reveals New}

\section{Dual Fluorescent Dyes and Unusually Bright Pyridine Derivatives}

\author{
Haishi Cao, Virginia Chang, Randy Hernandez, Michael D. Heagy* \\ Department of Chemistry, New Mexico Institute of Mining \& Technology \\ Socorro, NM 87801
}

Supplementary Table of Contents

Pages

I Absorption spectra for compounds 10, 13, 24, 27, 28

.........S1-S3

II. Experimental section synthetic procedures for compounds 1-42. S4-S18

I. Absorption Spectra

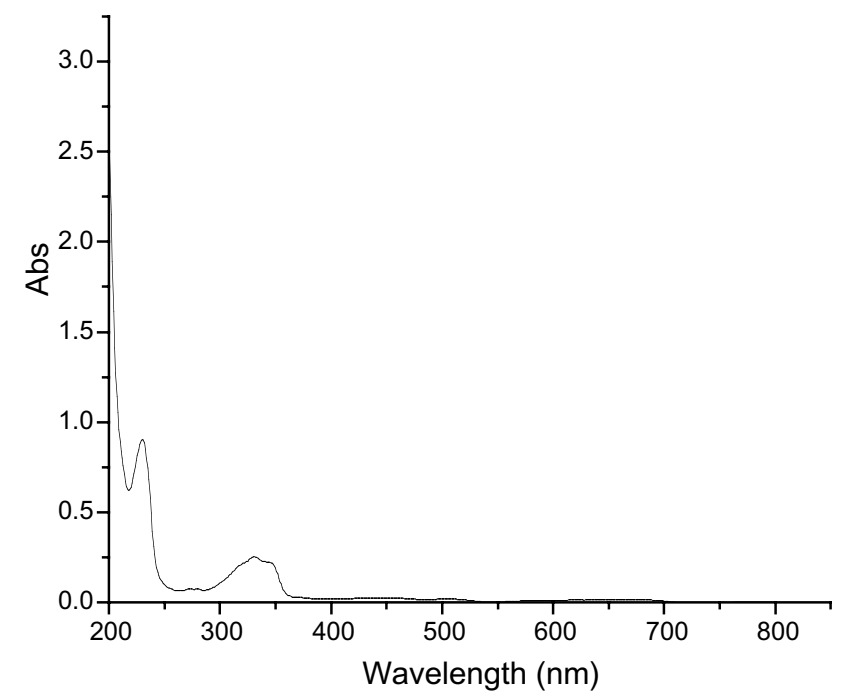

Figure S1: Compound $10\left(2.33 \times 10^{-5} \mathrm{M}\right)$ in acetonitrile. 


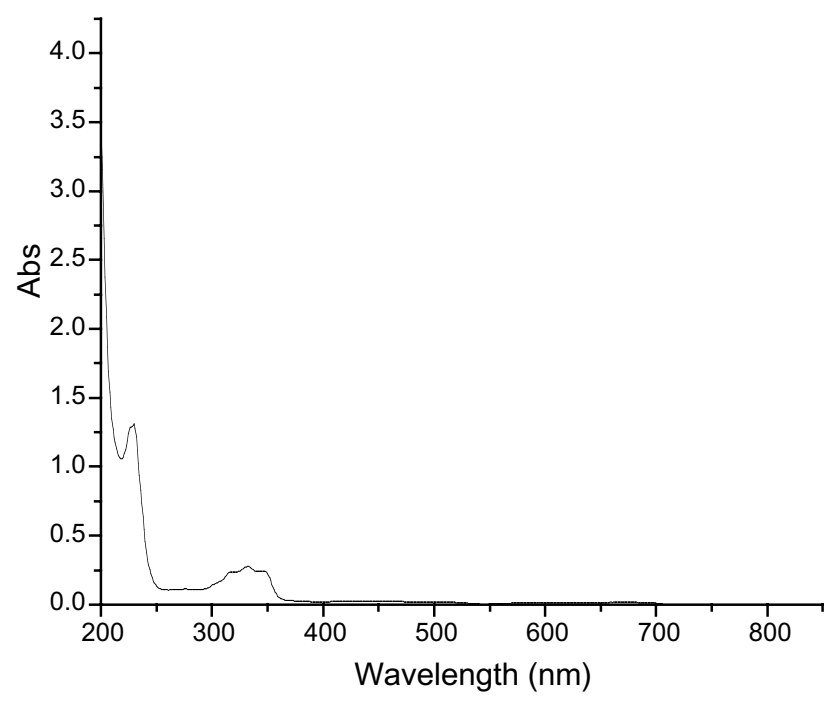

Figure S2: Compound $13\left(2.0 \times 10^{-5} \mathrm{M}\right)$ in acetonitrile.

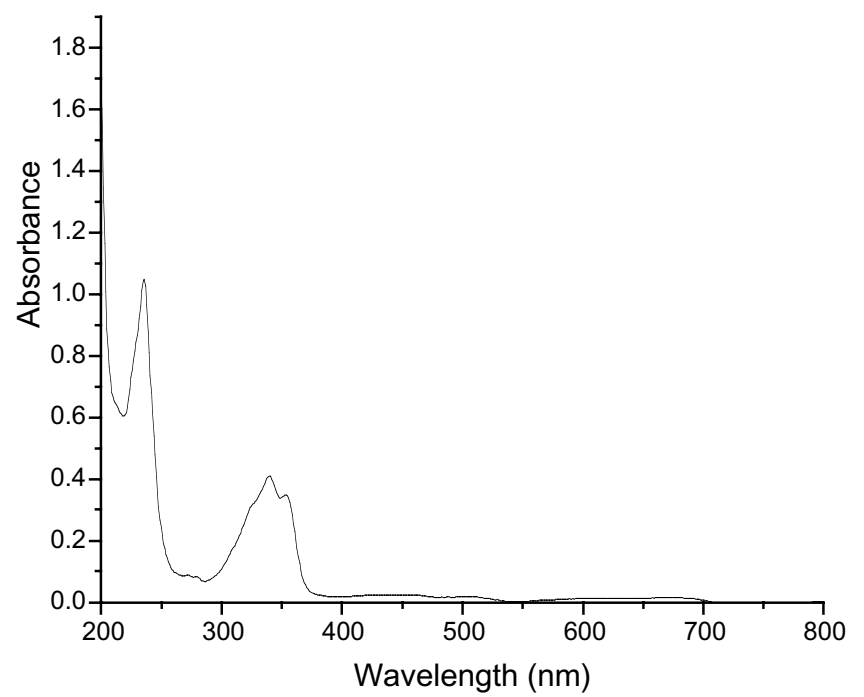

Figure S3. Compound $24\left(3.1 \times 10^{-5} \mathrm{M}\right)$ in acetonitrile. 


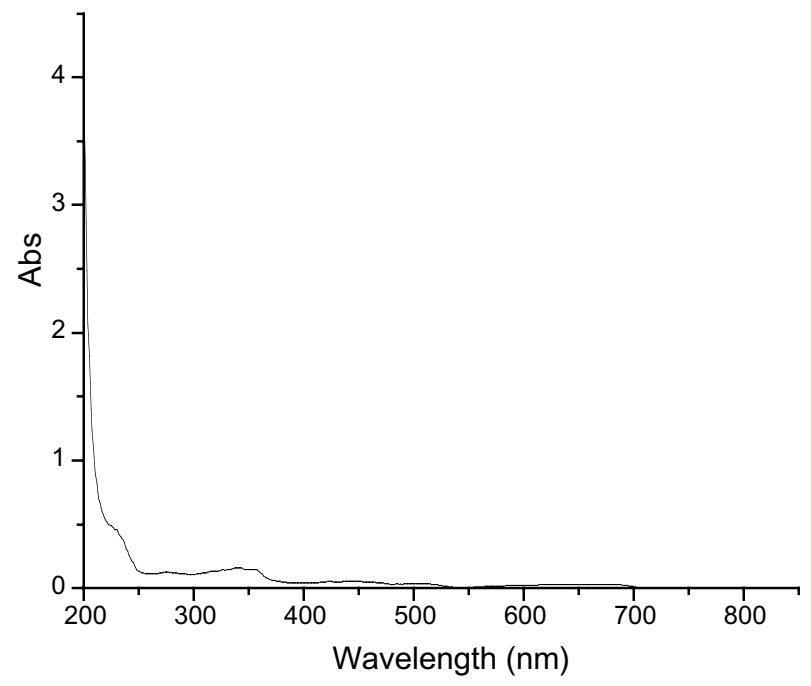

Figure S4. Compound $27\left(4.9 \times 10^{-6} \mathrm{M}\right)$ in acetonitrile

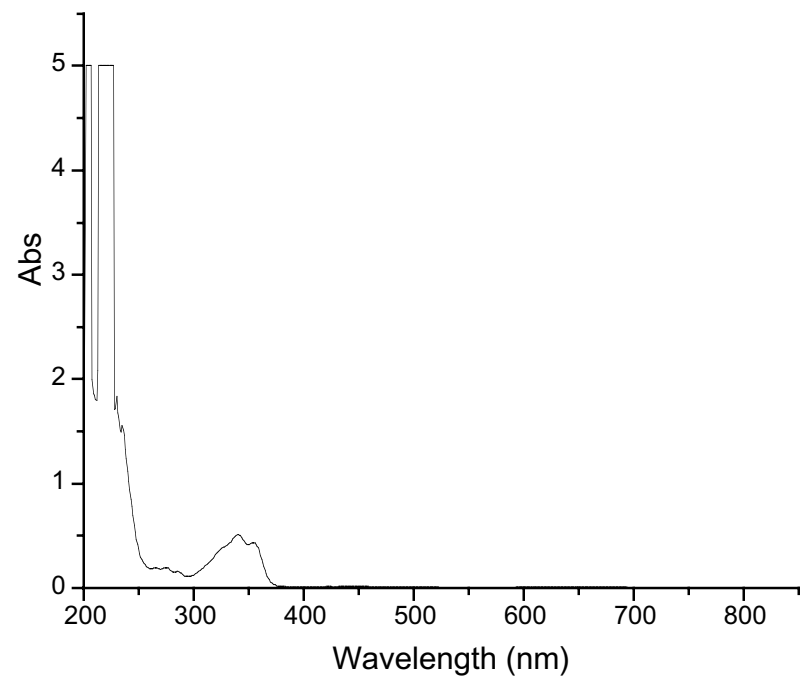

Figure S5. Compound $28\left(7.8 \times 10^{-5} \mathrm{M}\right)$ in acetonitrile 
II. Synthetic procedures

Synthesis for 1 to 14: 1,8-naphthalenedicarboxylic anhydride $(0.30 \mathrm{mmol})$, arylamine $(0.25 \mathrm{mmol}), 4 \AA$ molecular sieves, $30 \mathrm{mg} \mathrm{Zn}(\mathrm{OAc})_{2}$ and $25 \mathrm{ml}$ of pyridine were placed in a 50ml round-bottom flask, equipped with a Dean-Stark receiver and condenser. The reaction mixture was allowed to reflux for 8 hours. Pyridine was removed by distillation and replaced with acetone to precipitate a powder substance. The acetone solution was concentrated using a rotary evaporator and the crude solid was purified using silica gel preparative layer chromatography (PLC). The reaction typically afforded a white powder.

$N$-phenyl-1,8-naphthalimide (1) $\mathrm{mp} 191-193{ }^{\circ} \mathrm{C} ;{ }^{1} \mathrm{H}$ NMR $\left(\mathrm{CDCl}_{3}\right) \delta 8.66(\mathrm{~d}, J=3.6$ $\mathrm{Hz}, 2 \mathrm{H}), 8.26(\mathrm{~d}, J=3.1 \mathrm{~Hz}, 2 \mathrm{H}), 7.80(\mathrm{t}, J=3.9 \mathrm{~Hz}, 2 \mathrm{H}), 7.56(\mathrm{~m}, J=5.2 \mathrm{~Hz}, 3 \mathrm{H}), 7.31$ $(\mathrm{m}, J=4.8 \mathrm{~Hz}, 2 \mathrm{H}) ;{ }^{13} \mathrm{C} \mathrm{NMR}\left(\mathrm{CDCl}_{3}\right) \delta 164.4,135.8,134.3,131.8,131.6,129.5,128.7$, 127.1, 122.9. IR 1358, $1660 \mathrm{~cm}^{-1}$. Anal. Calcd for $\mathrm{C}_{18} \mathrm{H}_{11} \mathrm{NO}_{2}$ : C, 79.12; $\mathrm{H}, 4.03 ; \mathrm{N}$, 5.13. Found: C, 78.83; H, 4.03; N, 4.90.

$N$-(2'-pyridyl)-1,8-naphthalimide (2) $\mathrm{mp} 242-245{ }^{\circ} \mathrm{C} ;{ }^{1} \mathrm{H} \mathrm{NMR}\left(\mathrm{CDCl}_{3}\right) \delta 8.72(\mathrm{~d}, J=$ $2.4 \mathrm{~Hz}, 1 \mathrm{H}), 8.64(\mathrm{~d}, J=2.9 \mathrm{~Hz}, 2 \mathrm{H}), 8.28(\mathrm{~d}, J=2.9 \mathrm{~Hz}, 2 \mathrm{H}), 7.93(\mathrm{t}, J=3.4 \mathrm{~Hz}, 1 \mathrm{H})$, $7.78(\mathrm{t}, J=3.8 \mathrm{~Hz}, 2 \mathrm{H}), 7.42(\mathrm{~m}, J=4.9 \mathrm{~Hz}, 2 \mathrm{H}) ;{ }^{13} \mathrm{C} \mathrm{NMR}\left(\mathrm{CDCl}_{3}\right) \delta 164.3,150.1$, 149.7, 138.6, 134.6, 131.9, 131.6, 128.8, 127.1, 124.3, 124.1, 122.8. IR 1353, 1661, 1703 $\mathrm{cm}^{-1}$. Anal. Calcd for $\mathrm{C}_{17} \mathrm{H}_{10} \mathrm{~N}_{2} \mathrm{O}_{2}: \mathrm{C}, 74.45 ; \mathrm{H}, 3.65 ; \mathrm{N}, 10.28$. Found: $\mathrm{C}, 74.12 ; \mathrm{H}$, $3.66 ; \mathrm{N}, 10.23$. 
$\boldsymbol{N}$-(3'-pyridyl)-1,8-naphthalimide (3) $\mathrm{mp} 222-225{ }^{\circ} \mathrm{C} ;{ }^{1} \mathrm{H}$ NMR $\left(\mathrm{CDCl}_{3}\right) \delta 8.68(\mathrm{~m}, J=$ $7.4 \mathrm{~Hz}, 4 \mathrm{H}), 8.30(\mathrm{~d}, J=2.9 \mathrm{~Hz}, 2 \mathrm{H}), 7.82(\mathrm{~d}, J=3.9 \mathrm{~Hz}, 2 \mathrm{H}), 7.69(\mathrm{~d}, J=3.3 \mathrm{~Hz}, 1 \mathrm{H})$, $7.51(\mathrm{t}, J=3.6 \mathrm{~Hz}, 1 \mathrm{H}) ;{ }^{13} \mathrm{C} \mathrm{NMR}\left(\mathrm{CDCl}_{3}\right) \delta 164.2,150.0,149.9,149.6,136.6,134.7$, 132.2, 131.9, 128.7, 127.2, 123.9, 122.5. IR 1354, 1663, $1708 \mathrm{~cm}^{-1}$. Anal. Calcd for $\mathrm{C}_{17} \mathrm{H}_{10} \mathrm{~N}_{2} \mathrm{O}_{2}$ : C, 74.45; H, 3.65; N, 10.28. Found: C, 74.61; H, 3.64; N, 10.21.

$\boldsymbol{N}$-(4'-pyridyl)-1,8-naphthalimide (4) $\mathrm{mp} 308-310{ }^{\circ} \mathrm{C} ;{ }^{1} \mathrm{H}$ NMR $\left(\mathrm{CDCl}_{3}\right) \delta 8.85$ (d, $J=$ $2.4 \mathrm{~Hz}, 2 \mathrm{H}), 8.64(\mathrm{~d}, J=2.9 \mathrm{~Hz}, 2 \mathrm{H}), 8.29(\mathrm{~d}, J=2.9 \mathrm{~Hz}, 2 \mathrm{H}), 7.81(\mathrm{t}, J=3.4 \mathrm{~Hz}, 2 \mathrm{H})$, $7.31(\mathrm{~d}, J=2.5 \mathrm{~Hz}, 2 \mathrm{H}) ;{ }^{13} \mathrm{C}$ NMR $\left(\mathrm{CDCl}_{3}\right) \delta 163.6,151.2,143.4,134.8,132.0,131.9$, 127.2, 124.2, 124.1, 122.4. IR 1348, 1665, $1704 \mathrm{~cm}^{-1}$. Anal. Calcd for $\mathrm{C}_{17} \mathrm{H}_{10} \mathrm{~N}_{2} \mathrm{O}_{2}: \mathrm{C}$, 74.45; H, 3.65; N, 10.28. Found: C, 74.51; H, 3.68; N, 10.14.

$N$-(2'-pyrimidinyl)-1,8-naphthalimide (5) $\mathrm{mp} 298-302{ }^{\circ} \mathrm{C} ;{ }^{1} \mathrm{H}$ NMR $\left(\mathrm{CDCl}_{3}\right) \delta 9.00(\mathrm{~d}$, $J=2.9 \mathrm{~Hz}, 2 \mathrm{H}), 8.64(\mathrm{~d}, J=3.1 \mathrm{~Hz}, 2 \mathrm{H}), 8.29(\mathrm{~d}, J=3.3 \mathrm{~Hz}, 2 \mathrm{H}), 7.84(\mathrm{t}, J=3.9 \mathrm{~Hz}$, 2H), $7.50(\mathrm{t}, J=3.3 \mathrm{~Hz}, 1 \mathrm{H}) ;{ }^{13} \mathrm{C} \mathrm{NMR}\left(\mathrm{CDCl}_{3}\right) \delta 164.0,159.6,158.5,156.9,134.8$, 131.9, 131.7, 128.8, 127.1, 122.5, 121.1. IR 1357, 1663, $1703 \mathrm{~cm}^{-1}$. Anal. Calcd for $\mathrm{C}_{16} \mathrm{H}_{9} \mathrm{~N}_{3} \mathrm{O}_{2}$ : C, 69.82; H, 3.27; N, 15.27. Found: C, 69.63; H, 3.40; N, 15.49.

$\boldsymbol{N}$-(4'-pyrimidinyl)-1,8-naphthalimide (6) $\mathrm{mp} 301-303{ }^{\circ} \mathrm{C} ;{ }^{1} \mathrm{H}$ NMR $\left(\mathrm{CDCl}_{3}\right) \delta 8.64$ (m, $J=5.9 \mathrm{~Hz}, 3 \mathrm{H}), 8.33(\mathrm{~d}, J=3.1 \mathrm{~Hz}, 2 \mathrm{H}), 8.23(\mathrm{~d}, J=2.9 \mathrm{~Hz}, 1 \mathrm{H}), 7.85(\mathrm{t}, J=3.9 \mathrm{~Hz}$, 2H), $6.42(\mathrm{~d}, J=3.3 \mathrm{~Hz}, 1 \mathrm{H}) ;{ }^{13} \mathrm{C} \mathrm{NMR}\left(\mathrm{CDCl}_{3}\right) \delta 162.0,160.6,158.9,158.5,156.0$, 135.4, 133.5, 130.2, 127.6, 118.9, 105.2. IR 1357, 1663, $1703 \mathrm{~cm}^{-1}$. Anal. Calcd for $\mathrm{C}_{16} \mathrm{H}_{9} \mathrm{~N}_{3} \mathrm{O}_{2}$ : C, 69.82; H, 3.27; N, 15.27. Found: $\mathrm{C}, 70.02 ; \mathrm{H}, 3.21 ; \mathrm{N}, 15.34$. 
$N$-(3'-quinolyl)-1,8-naphthalimide (7) $\mathrm{mp} 228-231{ }^{\circ} \mathrm{C} ;{ }^{1} \mathrm{H} \mathrm{NMR}\left(\mathrm{CDCl}_{3}\right) \delta 8.85$ (s, $1 \mathrm{H}), 8.70(\mathrm{~d}, J=2.7 \mathrm{~Hz}, 2 \mathrm{H}), 8.33(\mathrm{~d}, J=2.5 \mathrm{~Hz}, 2 \mathrm{H}), 8.22(\mathrm{~d}, J=2.8 \mathrm{~Hz}, 2 \mathrm{H}), 7.90(\mathrm{~m}$, $J=6.9 \mathrm{~Hz}, 4 \mathrm{H}), 7.63(\mathrm{t}, J=3.3 \mathrm{~Hz}, 1 \mathrm{H}) ;{ }^{13} \mathrm{C} \mathrm{NMR}\left(\mathrm{CDCl}_{3}\right) \delta 164.5,150.3,147.3$, $135.8,134.8,132.0,131.9,130.3,129.5,129.0,128.7,128.2,128.1,127.5,127.2,122.6$. IR 1355, 1702, $2359 \mathrm{~cm}^{-1}$. Anal. Calcd for $\mathrm{C}_{21} \mathrm{H}_{12} \mathrm{~N}_{2} \mathrm{O}_{2}: \mathrm{C}, 77.78 ; \mathrm{H}, 3.70 ; \mathrm{N}, 8.64$. Found: C, 77.60; H, 3.81; N, 8.48.

$N$-(2'-methoxy phenyl)-1,8-naphthalimide (8) mp 245-248 ${ }^{\circ} \mathrm{C} ;{ }^{1} \mathrm{H}$ NMR $\left(\mathrm{CDCl}_{3}\right) \delta$ $8.68(\mathrm{~d}, J=2.9 \mathrm{~Hz}, 2 \mathrm{H}), 8.64(\mathrm{~d}, J=3.1 \mathrm{~Hz}, 2 \mathrm{H}), 7.79(\mathrm{t}, J=4.6 \mathrm{~Hz}, 2 \mathrm{H}), 7.46(\mathrm{t}, J=3.9$ $\mathrm{Hz}, 1 \mathrm{H}), 7.28(\mathrm{~d}, J=2.7 \mathrm{~Hz}, 1 \mathrm{H}), 7.12(\mathrm{t}, J=3.9 \mathrm{~Hz}, 1 \mathrm{H}), 3.83(\mathrm{~s}, 3 \mathrm{H}) ;{ }^{13} \mathrm{C} \mathrm{NMR}$ $\left(\mathrm{CDCl}_{3}\right) \delta 164.1,155.1,134.2,131.9,131.6,128.8,130.3,130.0,127.4,124.3,123.1$ 121.1, 112.1, 55.9. IR 1661, $1703 \mathrm{~cm}^{-1}$. Anal. Calcd for $\mathrm{C}_{19} \mathrm{H}_{13} \mathrm{NO}_{3}$ : C, 75.25; $\mathrm{H}, 4.29$; N, 4.62. Found: C, 74.77; H, 4.30; N, 4.72.

$N$-(3'-methoxy phenyl)-1,8-naphthalimide (9) mp 225-228 ${ }^{\circ} \mathrm{C} ;{ }^{1} \mathrm{H}$ NMR $\left(\mathrm{CDCl}_{3}\right) \delta$ $8.67(\mathrm{~d}, J=2.1 \mathrm{~Hz}, 2 \mathrm{H}), 8.28(\mathrm{~d}, J=2.2 \mathrm{~Hz}, 2 \mathrm{H}), 7.82(\mathrm{t}, J=2.9 \mathrm{~Hz}, 2 \mathrm{H}), 7.46(\mathrm{t}, J=2.9$ $\mathrm{Hz}, 1 \mathrm{H}), 7.08(\mathrm{~d}, J=2.2 \mathrm{~Hz}, 1 \mathrm{H}), 6.93(\mathrm{t}, J=4.1 \mathrm{~Hz}, 1 \mathrm{H}), 3.89(\mathrm{~s}, 3 \mathrm{H}) ;{ }^{13} \mathrm{C} \mathrm{NMR}$ $\left(\mathrm{CDCl}_{3}\right) \delta 164.3,160.5,136.6,134.3,131.8,131.7,130.3,130.1,127.1,122.9,120.9$ 114.9, 114.3, 55.4. IR 1357, 1667, $1706 \mathrm{~cm}^{-1}$. Anal. Calcd for $\mathrm{C}_{19} \mathrm{H}_{13} \mathrm{NO}_{3}: \mathrm{C}, 75.25 ; \mathrm{H}$, 4.29; N, 4.62. Found: C, 75.07; H, 4.37; N, 4.29. 
$N$-(4'-methoxy phenyl)-1,8-naphthalimide (10) mp 279-281 ${ }^{\circ} \mathrm{C} ;{ }^{1} \mathrm{H}$ NMR (DMSO) $\delta$ $8.51(\mathrm{~m}, J=2.7 \mathrm{~Hz}, 4 \mathrm{H}), 7.92(\mathrm{t}, J=3.2 \mathrm{~Hz}, 2 \mathrm{H}), 7.31(\mathrm{~d}, J=2.5 \mathrm{~Hz}, 2 \mathrm{H}), 7.07(\mathrm{~d}, J=$ $2.6 \mathrm{~Hz}, 2 \mathrm{H}), 3.98$ (s, 3H); ${ }^{13} \mathrm{C}$ NMR (DMSO) $\delta 164.3,159.4,134.9,132.0,131.2,130.6$, 129.0, 127.8, 123.2, 114.7, 55.9. IR 1376, 1669, $1701 \mathrm{~cm}^{-1}$. Anal. Calcd for $\mathrm{C}_{19} \mathrm{H}_{13} \mathrm{NO}_{3}$ : C, 75.25; H, 4.29; N, 4.62. Found: C, 75.15; H, 4.43; N, 4.57.

$N$-(3',5'-dimethoxy phenyl)-1,8-naphthalimide (11) mp 212-216 ${ }^{\circ} \mathrm{C} ;{ }^{1} \mathrm{H}$ NMR $\left(\mathrm{CDCl}_{3}\right)$ $\delta 8.73(\mathrm{~d}, J=2.5 \mathrm{~Hz}, 2 \mathrm{H}), 8.27(\mathrm{~d}, J=2.7 \mathrm{~Hz}, 2 \mathrm{H}), 7.80(\mathrm{t}, J=3.1 \mathrm{~Hz}, 2 \mathrm{H}), 6.57(\mathrm{~s}, 1 \mathrm{H})$, $6.47(\mathrm{~d}, J=2.2 \mathrm{~Hz}, 2 \mathrm{H}), 3.91(\mathrm{~s}, 6 \mathrm{H}) ;{ }^{13} \mathrm{C} \mathrm{NMR}\left(\mathrm{CDCl}_{3}\right) \delta 164.3,161.3,137.2,134.4$, 131.8, 131.6, 127.1, 122.8, 106.9, 101.4, 55.5. IR 1379, 1663, $1707 \mathrm{~cm}^{-1}$. Anal. Calcd for $\mathrm{C}_{20} \mathrm{H}_{15} \mathrm{NO}_{4}: \mathrm{C}, 72.07 ; \mathrm{H}, 4.50 ; \mathrm{N}, 4.20$. Found: C, 71.89; H, 4.58; N, 4.10.

$N$-(2'-thiazolyl)-1,8-naphthalimide (12) mp 308-312 ${ }^{\circ} \mathrm{C} ;{ }^{1} \mathrm{H}$ NMR $\left(\mathrm{CDCl}_{3}\right) \delta 8.67(\mathrm{~d}, J$ $=1.7 \mathrm{~Hz}, 2 \mathrm{H}), 8.36(\mathrm{t}, J=1.9 \mathrm{~Hz}, 2 \mathrm{H}), 7.93(\mathrm{~d}, J=1.2 \mathrm{~Hz}, 1 \mathrm{H}), 7.83(\mathrm{t}, J=2.6 \mathrm{~Hz}, 2 \mathrm{H})$, $7.61(\mathrm{~d}, J=1.3 \mathrm{~Hz}, 1 \mathrm{H}) ;{ }^{13} \mathrm{C} \mathrm{NMR}\left(\mathrm{CDCl}_{3}\right) \delta 164.0,155.4,141.4,135.0,132.1,131.9$, 129.2, 127.2, 122.5, 122.3. IR 1342, $1680,1709 \mathrm{~cm}^{-1}$. Anal. Calcd for $\mathrm{C}_{15} \mathrm{H}_{8} \mathrm{SN}_{2} \mathrm{O}_{2}$ : C, 68.13; H, 3.79; N, 4.42. Found: C, 68.23; H, 3.81; N, 4.38.

$\boldsymbol{N}$-(6'-quinolyl)-1,8-naphthalimide (13) mp 217-219 ${ }^{\circ} \mathrm{C} ;{ }^{1} \mathrm{H}$ NMR $\left(\mathrm{CDCl}_{3}\right) \delta 8.99$ (s, 1H), $8.68(\mathrm{~d}, J=2.3 \mathrm{~Hz}, 2 \mathrm{H}), 8.31(\mathrm{~m}, J=6.5 \mathrm{~Hz}, 4 \mathrm{H}), 7.84(\mathrm{~m}, J=4.8 \mathrm{~Hz}, 3 \mathrm{H}), 7.66(\mathrm{~d}$, $J=2.9 \mathrm{~Hz},, 4 \mathrm{H}), 7.47(\mathrm{t}, J=3.3 \mathrm{~Hz}, 1 \mathrm{H}) ;{ }^{13} \mathrm{C} \mathrm{NMR}\left(\mathrm{CDCl}_{3}\right) \delta 164.5,151.3,148.0$, 136.4, 134.6, 133.5, 131.8, 131.0, 130.1, 129.0, 127.9, 127.2, 122.8, 121.6. IR 1376, 
1655, $1704 \mathrm{~cm}^{-1}$. Anal. Calcd for $\mathrm{C}_{21} \mathrm{H}_{12} \mathrm{~N}_{2} \mathrm{O}_{2}$ : C, 77.78; H, 3.70; N, 8.64. Found: $\mathrm{C}$, $78.03 ; \mathrm{H}, 3.34 ; \mathrm{N}, 8.52$.

$\boldsymbol{N}$-(2'-naphthyl)-1,8-naphthalimide (14) $\mathrm{mp} 203-206{ }^{\circ} \mathrm{C} ;{ }^{1} \mathrm{H}$ NMR $\left(\mathrm{CDCl}_{3}\right) \delta 8.68(\mathrm{~d}, J$ $=2.2 \mathrm{~Hz}, 2 \mathrm{H}), 8.29(\mathrm{~d}, J=2.3 \mathrm{~Hz}, 2 \mathrm{H}), 8.02(\mathrm{~d}, J=2.1 \mathrm{~Hz}, 1 \mathrm{H}), 7.91(\mathrm{~m}, J=6.8 \mathrm{~Hz}$, $5 \mathrm{H}), 7.55(\mathrm{~m}, J=3.6 \mathrm{~Hz}, 2 \mathrm{H}), 7.42(\mathrm{~d}, J=2.7 \mathrm{~Hz}, 1 \mathrm{H}) ;{ }^{13} \mathrm{C} \mathrm{NMR}\left(\mathrm{CDCl}_{3}\right) \delta 164.6$, $134.4,133.7,133.2,132.9,13.1 .8,131.7,129.4,128.7,128.3,127.9,127.8,127.1,127.6$, 126.5, 126.3, 122.9. IR 1361, 1669, $1702 \mathrm{~cm}^{-1}$. Anal. Calcd for $\mathrm{C}_{22} \mathrm{H}_{13} \mathrm{NO}_{2}: \mathrm{C}, 81.73 ; \mathrm{H}$, 4.02; N, 4.33. Found: C, 81.59; H, 4.03; N,4.20.

Synthesis for 15 to 28: 4-Bromo-1,8-naphthalenedicarboxylic anhydride $(0.30 \mathrm{mmol})$, arylamine $(0.25 \mathrm{mmol}), 4 \AA$ molecular sieves, $30 \mathrm{mg} \mathrm{Zn}(\mathrm{OAc})_{2}$ and $25 \mathrm{ml}$ of pyridine were placed in a 50ml round-bottom flask, equipped with a Dean-Stark receiver and condenser. The reaction mixture was allowed to reflux for 8 hours. Pyridine was removed by distillation and replaced with acetone. The acetone solution was concentrated using a rotary evaporator and the crude solid was purified using silica gel PLC. The reaction afforded white powder.

4-Bromo- $\boldsymbol{N}$-phenyl-1,8-naphthalimide (15) mp 239-242 ${ }^{\circ} \mathrm{C} ;{ }^{1} \mathrm{H}$ NMR $\left(\mathrm{CDCl}_{3}\right) \delta 8.69$ $(\mathrm{m}, J=6.6 \mathrm{~Hz}, 2 \mathrm{H}), 8.44(\mathrm{~d}, J=3.1 \mathrm{~Hz}, 1 \mathrm{H}), 8.06(\mathrm{~d}, J=3.2 \mathrm{~Hz}, 1 \mathrm{H}), 7.86(\mathrm{t}, J=4.2 \mathrm{~Hz}$, 1H), $7.58(\mathrm{~m}, J=6.3 \mathrm{~Hz}, 4 \mathrm{H}), 7.33(\mathrm{~d}, J=3.9 \mathrm{~Hz}, 1 \mathrm{H}) ;{ }^{13} \mathrm{C} \mathrm{NMR}\left(\mathrm{CDCl}_{3}\right) \delta 163.8$, $135.2,133.7,132.5,131.6,131.3,130.8,130.7,129.5,129.4,128.9,128.6,128.3,123.3$, 
122.3. IR 1367, $1662 \mathrm{~cm}^{-1}$. Anal. Calcd for $\mathrm{C}_{18} \mathrm{H}_{10} \mathrm{BrNO}_{2}$ : C, 61.36; H, 2.84; N, 3.98 . Found: $\mathrm{C}, 61.11 ; \mathrm{H}, 2.70 ; \mathrm{N}, 3.81$.

4-Bromo- $N$-(2'-pyridyl)-1,8-naphthalimide (16) mp 265-269 ${ }^{\circ} \mathrm{C}$; ${ }^{1} \mathrm{H}$ NMR $\left(\mathrm{CDCl}_{3}\right) \delta$ $8.69(\mathrm{~m}, J=6.9 \mathrm{~Hz}, 3 \mathrm{H}), 8.46(\mathrm{~d}, J=2.3 \mathrm{~Hz}, 1 \mathrm{H}), 8.08(\mathrm{~d}, J=2.3 \mathrm{~Hz}, 1 \mathrm{H}), 7.96(\mathrm{~m}, J=$ $6.8 \mathrm{~Hz}, 2 \mathrm{H}), 7.42(\mathrm{~m}, J=5.2 \mathrm{~Hz}, 2 \mathrm{H}) ;{ }^{13} \mathrm{C} \mathrm{NMR}\left(\mathrm{CDCl}_{3}\right) \delta 163.7,150.1,149.3,138.5$, $133.9,132.5,131.6,131.3,131.0,130.9,129.5,128.2,124.3,124.2,123.1,122.2$. IR $1369,1670 \mathrm{~cm}^{-1}$. Anal. Calcd for $\mathrm{C}_{17} \mathrm{H}_{9} \mathrm{BrN}_{2} \mathrm{O}_{2}: \mathrm{C}, 57.79 ; \mathrm{H}, 2.55 ; \mathrm{N}, 7.93$. Found: $\mathrm{C}$, $57.93 ; \mathrm{H}, 2.43 ; \mathrm{N}, 7.81$.

4-Bromo- $N$-(3'-pyridyl)-1,8-naphthalimide (17) $\mathrm{mp}$ 247-251 ${ }^{\circ} \mathrm{C}$; ${ }^{1} \mathrm{H}$ NMR (Acetone$\left.\mathrm{D}_{6}\right) \delta 8.71(\mathrm{~m}, J=3.2 \mathrm{~Hz}, 4 \mathrm{H}), 8.46(\mathrm{~d}, J=2.2 \mathrm{~Hz}, 1 \mathrm{H}), 8.29(\mathrm{~d}, J=2.4 \mathrm{~Hz}, 1 \mathrm{H}), 8.07(\mathrm{t}$, $J=3.3 \mathrm{~Hz}, 1 \mathrm{H}), 7.91(\mathrm{t}, J=2.8 \mathrm{~Hz}, 1 \mathrm{H}), 7.61(\mathrm{t}, J=2.9 \mathrm{~Hz}, 1 \mathrm{H}) ;{ }^{13} \mathrm{C}$ NMR (AcetoneD ${ }_{6} \delta 150.2,149.3,140.5,136.8,133.2,132.8,131.9,131.5,131.2,130.7,129.7,129.5$, 128.7, 123.8, 123.1. IR 1662, $1710 \mathrm{~cm}^{-1}$. Anal. Calcd for $\mathrm{C}_{17} \mathrm{H}_{9} \mathrm{BrN}_{2} \mathrm{O}_{2}: \mathrm{C}, 57.79 ; \mathrm{H}$, 2.55; N, 7.93. Found: C, 57.92; H, 2.49; N, 7.72.

4-Bromo- $N$-(4'-pyridyl)-1,8-naphthalimide (18) $\mathrm{mp}$ 281-283 ${ }^{\circ} \mathrm{C} ;{ }^{1} \mathrm{H}$ NMR (DMSO) $\delta$ $8.79(\mathrm{~d}, J=2.1 \mathrm{~Hz}, 2 \mathrm{H}), 8.65(\mathrm{~d}, J=4.4 \mathrm{~Hz}, 2 \mathrm{H}), 8.39(\mathrm{~d}, J=2.4 \mathrm{~Hz}, 1 \mathrm{H}), 8.30(\mathrm{t}, J=$ $2.4 \mathrm{~Hz}, 1 \mathrm{H}), 8.05(\mathrm{t}, J=3.5 \mathrm{~Hz}, 1 \mathrm{H}), 7.53(\mathrm{~d}, J=2.4 \mathrm{~Hz}, 2 \mathrm{H}) ;{ }^{13} \mathrm{C}$ NMR (DMSO) $\delta$ $163.3,151.2,144.3,142.2,134.8,133.6,132.3,132.1,131.7,130.6,130.1,129.5,129.4$, 125.1, 123.7, 122.9. IR 1363, 1672, $1711 \mathrm{~cm}^{-1}$. Anal. Calcd for $\mathrm{C}_{17} \mathrm{H}_{9} \mathrm{BrN}_{2} \mathrm{O}_{2}$ : C, 57.79; H, 2.55; N, 7.93. Found: C, 57.52; H, 2.60; N, 7.86. 
4-Bromo- $N$-(2'-pyrimidinyl)-1,8-naphthalimide (19) $\mathrm{mp} \quad 277-279 \quad{ }^{\circ} \mathrm{C} ;{ }^{1} \mathrm{H} \quad \mathrm{NMR}$ $\left(\mathrm{CDCl}_{3}\right) \delta 8.99(\mathrm{~d}, J=2.4 \mathrm{~Hz}, 2 \mathrm{H}), 8.68(\mathrm{~m}, J=5.3 \mathrm{~Hz}, 2 \mathrm{H}), 8.46(\mathrm{~d}, J=2.5 \mathrm{~Hz}, 1 \mathrm{H})$, $8.09(\mathrm{~d}, J=2.4 \mathrm{~Hz}, 1 \mathrm{H}), 7.91(\mathrm{t}, J=3.4 \mathrm{~Hz}, 1 \mathrm{H}), 7.51(\mathrm{t}, J=3.2 \mathrm{~Hz}, 1 \mathrm{H}) ;{ }^{13} \mathrm{C} \mathrm{NMR}$ $\left(\mathrm{CDCl}_{3}\right) \delta 163.4,159.6,156.6,134.1,132.5,131.6,131.5,131.4,131.2,131.0,128.3$ 123.0, 122.1, 121.3. IR 1364, 1670, $1709 \mathrm{~cm}^{-1}$. Anal. Calcd for $\mathrm{C}_{16} \mathrm{H}_{8} \mathrm{BrN}_{3} \mathrm{O}_{2}$ : C, 54.23; H, 2.26; N, 11.86. Found: C, 54.56; H, 2.46; N, 11.77.

4-Bromo- $N$-(4'-pyrimidinyl)-1,8-naphthalimide (20) $\mathrm{mp} \quad 285-297 \quad{ }^{\circ} \mathrm{C} ;{ }^{1} \mathrm{H} \quad \mathrm{NMR}$ $\left(\mathrm{CDCl}_{3}\right) \delta 9.39(\mathrm{~s}, 1 \mathrm{H}), 9.01(\mathrm{~d}, J=1.9 \mathrm{~Hz}, 1 \mathrm{H}), 8.71(\mathrm{~m}, J=3.5 \mathrm{~Hz}, 2 \mathrm{H}), 8.47(\mathrm{~d}, J=$ $2.3 \mathrm{~Hz}, 1 \mathrm{H}), 8.11(\mathrm{~d}, J=2.3 \mathrm{~Hz}, 1 \mathrm{H}), 7.93(\mathrm{t}, J=2.8 \mathrm{~Hz}, 1 \mathrm{H}), 7.53(\mathrm{~d}, J=2.1 \mathrm{~Hz}, 1 \mathrm{H})$; ${ }^{13} \mathrm{C} \mathrm{NMR}\left(\mathrm{CDCl}_{3}\right) \delta 163.1,160.0,159.4,157.5,139.1,134.4,132.8,131.9,131.6,131.5$, 131.0, 128.4, 122.9, 121.9, 121.8. IR 1363, 1671, $1710 \mathrm{~cm}^{-1}$. Anal. Calcd for $\mathrm{C}_{16} \mathrm{H}_{8} \mathrm{BrN}_{3} \mathrm{O}_{2}$ : C, 54.23; H, 2.26; N, 11.86. Found: C, 54.46; H, 2.23; N, 11.96.

4-Bromo- $N$-(3'-quinolyl)-1,8-naphthalimide (21) mp 213-215 ${ }^{\circ} \mathrm{C} ;{ }^{1} \mathrm{H}$ NMR (DMSO) $\delta$ $8.94(\mathrm{~s}, 1 \mathrm{H}), 8.70(\mathrm{t}, J=4.2 \mathrm{~Hz}, 2 \mathrm{H}), 8.50(\mathrm{~d}, J=1.8 \mathrm{~Hz}, 1 \mathrm{H}), 8.44(\mathrm{~d}, J=2.4 \mathrm{~Hz}, 1 \mathrm{H})$, $8.33(\mathrm{~d}, J=2.4 \mathrm{~Hz}, 1 \mathrm{H}), 8.15(\mathrm{~m}, J=6.1 \mathrm{~Hz}, 3 \mathrm{H}), 7.91(\mathrm{t}, J=3.6 \mathrm{~Hz}, 1 \mathrm{H}), 7.72(\mathrm{t}, J=$ $3.3 \mathrm{~Hz}, 1 \mathrm{H}) ;{ }^{13} \mathrm{C}$ NMR (DMSO) $\delta 163.9,151.2,145.8,136.1,133.9,132.8,132.6,131.7$, 132.2, 131.2, 129.1, 128.8, 128.4, 128.0, 127.6, 123.9, 122.6, 112.5. IR 1361, 1665, 1703 $\mathrm{cm}^{-1}$. Anal. Calcd for $\mathrm{C}_{21} \mathrm{H}_{11} \mathrm{BrN}_{2} \mathrm{O}_{2}$ : C, 62.53; H, 2.73; N, 6.95. Found: C, 62.34; H, $2.76 ; \mathrm{N}, 6.84$. 
4-Bromo- $N$-(2'-methoxy phenyl)-1,8-naphthalimide (22) mp 237-240 ${ }^{\circ} \mathrm{C}$; ${ }^{1} \mathrm{H}$ NMR $\left(\mathrm{CDCl}_{3}\right) \delta 8.70(\mathrm{~d}, J=2.6 \mathrm{~Hz}, 1 \mathrm{H}), 8.62(\mathrm{~d}, J=2.6 \mathrm{~Hz}, 1 \mathrm{H}), 8.46(\mathrm{~d}, J=2.6 \mathrm{~Hz}, 1 \mathrm{H})$, $8.07(\mathrm{~d}, J=2.7 \mathrm{~Hz}, 1 \mathrm{H}), 7.89(\mathrm{t}, J=3.1 \mathrm{~Hz}, 1 \mathrm{H}), 7.47(\mathrm{t}, J=3.4 \mathrm{~Hz}, 1 \mathrm{H}), 7.25(\mathrm{t}, J=2.9$ $\mathrm{Hz}, 1 \mathrm{H}), 7.14(\mathrm{t}, J=3.9 \mathrm{~Hz}, 2 \mathrm{H}), 3.77(\mathrm{~s}, 3 \mathrm{H}) ;{ }^{13} \mathrm{C} \mathrm{NMR}\left(\mathrm{CDCl}_{3}\right) \delta 163.5,155.0,133.5$, $132.4,131.6,131.1,130.9,130.5,129.9,129.6,123.9,123.5,123.1,128.2,122.6,121.1$, 112.1, 55.9. IR 1370, 1665, $1708 \mathrm{~cm}^{-1}$. Anal. Calcd for $\mathrm{C}_{19} \mathrm{H}_{12} \mathrm{BrNO}_{3}$ : C, 59.69; H, 3.14; N, 3.66. Found: C, 59.45; H, 3.22; N, 3.62.

4-Bromo- $N$-(3'-methoxy phenyl)-1,8-naphthalimide (23) mp $234-237{ }^{\circ} \mathrm{C}$; ${ }^{1} \mathrm{H}$ NMR $\left(\mathrm{CDCl}_{3}\right) \delta 8.71(\mathrm{~d}, J=2.2 \mathrm{~Hz}, 2 \mathrm{H}), 8.64(\mathrm{~d}, J=2.2 \mathrm{~Hz}, 1 \mathrm{H}), 8.46(\mathrm{~d}, J=2.2 \mathrm{~Hz}, 1 \mathrm{H})$, $8.09(\mathrm{~d}, J=2.3 \mathrm{~Hz}, 1 \mathrm{H}), 7.91(\mathrm{t}, J=2.9 \mathrm{~Hz}, 1 \mathrm{H}), 7.48(\mathrm{~d}, J=2.8 \mathrm{~Hz}, 1 \mathrm{H}), 7.02(\mathrm{~d}, J=2.1$ $\mathrm{Hz}, 1 \mathrm{H}), 6.91(\mathrm{~d}, J=3.8 \mathrm{~Hz}, 2 \mathrm{H}), 3.83(\mathrm{~s}, 3 \mathrm{H}) ;{ }^{13} \mathrm{C} \mathrm{NMR}\left(\mathrm{CDCl}_{3}\right) \delta 163.8,160.5,136.2$, $133.8,132.6,131.7,131.3,130.9,130.8,130.2,129.5,128.3,123.3,122.4,120.8,115.0$, 114.2, 55.5. IR 1369, 1665, $1708 \mathrm{~cm}^{-1}$. Anal. Calcd for $\mathrm{C}_{19} \mathrm{H}_{12} \mathrm{BrNO}_{3}$ : C, 59.69; H, 3.14; N, 3.66. Found: C, 59.52; H, 3.21; N, 3.61.

4-Bromo- $\boldsymbol{N}$-(4'-methoxy phenyl)-1,8-naphthalimide (24) mp 227-229 ${ }^{\circ} \mathrm{C}$; ${ }^{1} \mathrm{H}$ NMR $\left(\mathrm{CDCl}_{3}\right) \delta 8.69(\mathrm{~d}, J=2.6 \mathrm{~Hz}, 1 \mathrm{H}), 8.64(\mathrm{~d}, J=2.8 \mathrm{~Hz}, 1 \mathrm{H}), 8.47(\mathrm{~d}, J=2.9 \mathrm{~Hz}, 1 \mathrm{H})$, $8.08(\mathrm{~d}, J=2.9 \mathrm{~Hz}, 1 \mathrm{H}), 7.91(\mathrm{t}, J=3.4 \mathrm{~Hz}, 1 \mathrm{H}), 7.21(\mathrm{~d}, J=3.1 \mathrm{~Hz}, 2 \mathrm{H}), 7.07(\mathrm{~d}, J=$ $2.9 \mathrm{~Hz}, 2 \mathrm{H}), 3.94(\mathrm{~s}, 3 \mathrm{H}) ;{ }^{13} \mathrm{C} \mathrm{NMR}\left(\mathrm{CDCl}_{3}\right) \delta 164.1,159.7,133.7,132.5,131.7,131.3$, $130.8,130.7,129.5,129.4,128.3,127.6,123.4,122.5,114.9,55.6$. IR 1366, 1659, 1707 $\mathrm{cm}^{-1}$. Anal. Calcd for $\mathrm{C}_{19} \mathrm{H}_{12} \mathrm{BrNO}_{3}$ : C, 59.69; H, 3.14; N, 3.66. Found: C, 59.77; H, $3.19 ; \mathrm{N}, 3.58$. 
4-Bromo- $N$-(3',5'-dimethoxy phenyl)-1,8-naphthalimide (25) mp $294-296 \quad{ }^{\circ} \mathrm{C}$; ${ }^{1} \mathrm{H}$ $\operatorname{NMR}\left(\mathrm{CDCl}_{3}\right) \delta 8.69(\mathrm{~d}, J=2.1 \mathrm{~Hz}, 1 \mathrm{H}), 8.62(\mathrm{~d}, J=2.2 \mathrm{~Hz}, 1 \mathrm{H}), 8.47(\mathrm{~d}, J=2.2 \mathrm{~Hz}$, $1 \mathrm{H}), 8.09(\mathrm{~d}, J=2.1 \mathrm{~Hz}, 1 \mathrm{H}), 7.91(\mathrm{t}, J=2.9 \mathrm{~Hz}, 1 \mathrm{H}), 6.59(\mathrm{~s}, 1 \mathrm{H}), 6.46(\mathrm{~s}, 2 \mathrm{H}), 3.85(\mathrm{~s}$, $6 \mathrm{H}) ;{ }^{13} \mathrm{C} \mathrm{NMR}\left(\mathrm{CDCl}_{3}\right) \delta 163.9,161.4,136.9,134.1,132.5,130.7,130.2,131.5,131.1$, 129.5, 128.2, 123.1, 122.2, 106.7, 101.5, 55.5. IR 1339, 1666, $1715 \mathrm{~cm}^{-1}$. Anal. Calcd for $\mathrm{C}_{20} \mathrm{H}_{14} \mathrm{BrNO}_{4}$ : C, 58.25; H, 3.40; N, 3.40. Found: C, 58.28; H, 3.49; N, 3.34.

4-Bromo- $N$-(2'-thiazolyl)-1,8-naphthalimide (26) mp 282-284 ${ }^{\circ} \mathrm{C} ;{ }^{1} \mathrm{H} \mathrm{NMR}\left(\mathrm{CDCl}_{3}\right) \delta$ $8.72(\mathrm{~m}, J=6.7 \mathrm{~Hz}, 2 \mathrm{H}), 8.48(\mathrm{~d}, J=2.2 \mathrm{~Hz}, 1 \mathrm{H}), 8.11(\mathrm{~d}, J=2.1 \mathrm{~Hz}, 1 \mathrm{H}), 7.93(\mathrm{~m}, J=$ $5.6 \mathrm{~Hz}, 2 \mathrm{H}), 7.62(\mathrm{~d}, J=2.1 \mathrm{~Hz}, 1 \mathrm{H}) ;{ }^{13} \mathrm{C} \mathrm{NMR}\left(\mathrm{CDCl}_{3}\right) \delta 163.2,141.5,134.4,133.0$, 132.0, 131.6, 131.5, 131.0, 129.5, 128.5, 128.4, 122.6, 122.3. IR 1342, $1664,1709 \mathrm{~cm}^{-1}$. Anal. Calcd for $\mathrm{C}_{15} \mathrm{H}_{7} \mathrm{BrSN}_{2} \mathrm{O}_{2}: \mathrm{C}, 50.14 ; \mathrm{H}, 1.95 ; \mathrm{N}, 7.80$. Found: C, 50.40; H, 1.85; N, 7.66 .

4-Bromo- $N$-(6'-quinolyl)-1,8-naphthalimide (27) mp 189-193 ${ }^{\circ} \mathrm{C} ;{ }^{1} \mathrm{H}$ NMR $\left(\mathrm{CDCl}_{3}\right) \delta$ $8.99(\mathrm{~d}, J=1.6 \mathrm{~Hz}, 1 \mathrm{H}), 8.70(\mathrm{~d}, J=2.3 \mathrm{~Hz}, 1 \mathrm{H}), 8.64(\mathrm{~d}, J=2.2 \mathrm{~Hz}, 1 \mathrm{H}), 8.46(\mathrm{~d}, J=$ $2.3 \mathrm{~Hz}, 1 \mathrm{H}), 8.25(\mathrm{~d}, J=2.3 \mathrm{~Hz}, 1 \mathrm{H}), 8.19(\mathrm{~d}, J=2.2 \mathrm{~Hz}, 1 \mathrm{H}), 8.11(\mathrm{~d}, J=2.3 \mathrm{~Hz}, 1 \mathrm{H})$, $7.91(\mathrm{~m}, J=5.8 \mathrm{~Hz}, 2 \mathrm{H}), 7.64(\mathrm{~d}, J=2.5 \mathrm{~Hz}, 1 \mathrm{H}), 7.47(\mathrm{t}, J=3.5 \mathrm{~Hz}, 1 \mathrm{H}) ;{ }^{13} \mathrm{C} \mathrm{NMR}$ $\left(\mathrm{CDCl}_{3}\right) \delta 163.9,151.4,148.0,136.4,133.9,133.1,132.6,131.8,131.4,131.1,130.9$ 130.0, 129.5, 128.5, 128.4, 127.8, 123.2, 122.8, 121.6. IR 1367, 1664, $1711 \mathrm{~cm}^{-1}$. Anal. Calcd for $\mathrm{C}_{21} \mathrm{H}_{11} \mathrm{BrN}_{2} \mathrm{O}_{2}$ : C, 62.53; H, 2.73; N, 6.95. Found: C, 62.26; H, 2.79; N, 6.78. 
4-Bromo- $N$-(2'-naphthyl)-1,8-naphthalimide (28) mp 196-199 ${ }^{\circ} \mathrm{C} ;{ }^{1} \mathrm{H} \mathrm{NMR}\left(\mathrm{CDCl}_{3}\right) \delta$ $8.72(\mathrm{~d}, J=2.2 \mathrm{~Hz}, 1 \mathrm{H}), 8.63(\mathrm{~d}, J=2.3 \mathrm{~Hz}, 1 \mathrm{H}), 8.47(\mathrm{~d}, J=2.3 \mathrm{~Hz}, 1 \mathrm{H}), 8.09(\mathrm{~d}, J=$ $2.3 \mathrm{~Hz}, 1 \mathrm{H}), 8.01(\mathrm{~d}, J=2.2 \mathrm{~Hz}, 1 \mathrm{H}), 7.91(\mathrm{~m}, J=6.8 \mathrm{~Hz}, 5 \mathrm{H}), 7.55(\mathrm{~m}, J=3.9 \mathrm{~Hz},, 2 \mathrm{H})$, $7.39(\mathrm{~d}, J=2.4 \mathrm{~Hz}, 1 \mathrm{H}) ;{ }^{13} \mathrm{C} \mathrm{NMR}\left(\mathrm{CDCl}_{3}\right) \delta 164.1,151.7,147.5,136.5,133.9,133.7$, $133.1,132.1,132.6,132.1,131.7,130.8,129.5,129.5,129.3,128.8,128.3,127.9,126.1$, 123.2, 122.1. IR 1367, 1663, $1712 \mathrm{~cm}^{-1}$. Anal. Calcd for $\mathrm{C}_{22} \mathrm{H}_{12} \mathrm{BrNO}_{2}: \mathrm{C}, 65.57 ; \mathrm{H}$, 2.99; N, 3.48. Found: C, 65.64; H, 3.05; N, 3.38.

Synthesis for 29 to 42: 4-Bromo-1,8-naphthalimide derivatives (compounds 15 to 28) (0.20 mmol), $15 \mathrm{mg}$ of $\mathrm{CuBr}$, and a 10:1 stoichiometric ratio of sodium methoxide in methanol. It was stirred and refluxed for 6 hours. Methanol was removed by distillation. Crude product was purified by silica gel PLC. The reaction afforded a tan or yellow powder

4-Methoxy- $N$-phenyl-1,8-naphthalimide (29) $\mathrm{mp} 235-239{ }^{\circ} \mathrm{C} ;{ }^{1} \mathrm{H} \mathrm{NMR}\left(\mathrm{CDCl}_{3}\right) \delta 8.61$ $(\mathrm{m}, J=5.3 \mathrm{~Hz}, 3 \mathrm{H}), 7.72(\mathrm{t}, J=3.1 \mathrm{~Hz}, 1 \mathrm{H}), 7.54(\mathrm{~m}, J=6.5 \mathrm{~Hz}, 3 \mathrm{H}), 7.33(\mathrm{~m}, J=5.2$ $\mathrm{Hz}, 2 \mathrm{H}), 7.09(\mathrm{~d}, J=2.8 \mathrm{~Hz}, 1 \mathrm{H}), 4.19(\mathrm{~s}, 3 \mathrm{H}) ;{ }^{13} \mathrm{C} \mathrm{NMR}\left(\mathrm{CDCl}_{3}\right) \delta 164.8,164.2,135.8$, $133.9,132.0,129.4,129.1,128.7,128.6,126.1,123.8,122.6,115.2,105.4,56.4$. IR 1363, 1655, $1697 \mathrm{~cm}^{-1}$. Anal. Calcd for $\mathrm{C}_{19} \mathrm{H}_{13} \mathrm{NO}_{3}: \mathrm{C}, 75.25 ; \mathrm{H}, 4.29 ; \mathrm{N}, 4.62$. Found: C, 75.28; H, 4.21; N, 4.52.

4-Methoxy-N-(2'-pyridyl)-1,8-naphthalimide (30) mp 223-227 ${ }^{\circ} \mathrm{C} ;{ }^{1} \mathrm{H} \mathrm{NMR}\left(\mathrm{CDCl}_{3}\right) \delta$ $8.74(\mathrm{~d}, J=2.2 \mathrm{~Hz}, 1 \mathrm{H}), 8.64(\mathrm{~d}, J=5.9 \mathrm{~Hz}, 3 \mathrm{H}), 7.92(\mathrm{t}, J=3.3 \mathrm{~Hz}, 1 \mathrm{H}), 7.73(\mathrm{t}, J=3.6$ 
$\mathrm{Hz}, 1 \mathrm{H}), 7.41(\mathrm{~m}, J=4.9 \mathrm{~Hz}, 2 \mathrm{H}), 7.09(\mathrm{~d}, J=2.4 \mathrm{~Hz}, 1 \mathrm{H}), 4.20(\mathrm{~s}, 3 \mathrm{H}) ;{ }^{13} \mathrm{C}$ NMR $\left(\mathrm{CDCl}_{3}\right) \delta 164.1,161.3,150.0,138.5,134.0,132.0,130.1,130.0,129.3,126.1,124.3$, 124.0, 123.8, 122.5, 115.1, 105.4, 56.4. IR 1360, 1668, $1697 \mathrm{~cm}^{-1}$. Anal. Calcd for $\mathrm{C}_{18} \mathrm{H}_{12} \mathrm{~N}_{2} \mathrm{O}_{3}$ : C, 71.05; H, 3.95; N, 9.21. Found: C, 70.75; H, 3.81; N, 9.07.

4-Methoxy- $N$-(3'-pyridyl)-1,8-naphthalimide (31) mp 204-206 ${ }^{\circ} \mathrm{C} ;{ }^{1} \mathrm{H}$ NMR (DMSO) $\delta 8.63(\mathrm{~m}, J=7.4 \mathrm{~Hz}, 5 \mathrm{H}), 7.94(\mathrm{~d}, J=3.9 \mathrm{~Hz}, 2 \mathrm{H}), 7.64(\mathrm{~s}, 1 \mathrm{H}), 7.39(\mathrm{~d}, J=2.8 \mathrm{~Hz}$, 1H), 4.15 (s, 3H); ${ }^{13} \mathrm{C}$ NMR (DMSO) $\delta 164.7,164.4,161.2,134.4,134.1,131.8,131.3$, 130.6, 129.2, 128.4, 127.1, 123.7, 122.6, 114.6, 106.9, 56.4. IR 1354, 1661, $1703 \mathrm{~cm}^{-1}$. Anal. Calcd for $\mathrm{C}_{18} \mathrm{H}_{12} \mathrm{~N}_{2} \mathrm{O}_{3}:$ C, 71.05; H, 3.95; N, 9.21. Found: C, 71.14; H, 4.04; N, 9.07 .

4-Methoxy- $N$-(4'-pyridyl)-1,8-naphthalimide (32) mp 262-265 ${ }^{\circ} \mathrm{C} ;{ }^{1} \mathrm{H} \mathrm{NMR}\left(\mathrm{CDCl}_{3}\right) \delta$ $8.82(\mathrm{~d}, J=2.2 \mathrm{~Hz}, 2 \mathrm{H}), 8.66(\mathrm{~m}, J=4.8 \mathrm{~Hz}, 3 \mathrm{H}), 7.78(\mathrm{t}, J=3.1 \mathrm{~Hz}, 1 \mathrm{H}), 7.32(\mathrm{~d}, J=$ $2.5 \mathrm{~Hz}, 2 \mathrm{H}), 7.11(\mathrm{~d}, J=2.4 \mathrm{~Hz}, 1 \mathrm{H}), 4.16(\mathrm{~s}, 3 \mathrm{H}) ;{ }^{13} \mathrm{C} \mathrm{NMR}\left(\mathrm{CDCl}_{3}\right) \delta 163.4,151.2$, 134.4, 132.0, 129.0, 126.2, 124.0, 105.3, 56.1. IR 1360, 1669, $1697 \mathrm{~cm}^{-1}$. Anal. Calcd for $\mathrm{C}_{18} \mathrm{H}_{12} \mathrm{~N}_{2} \mathrm{O}_{3}:$ C, 71.05; H, 3.95; N, 9.21. Found: C, 71.23; H, 4.01; N, 9.13.

4-Methoxy- $N$-(2'-pyrimidinyl)-1,8-naphthalimide (33) $\mathrm{mp}$ 265-267 ${ }^{\circ} \mathrm{C} ;{ }^{1} \mathrm{H}$ NMR $\left(\mathrm{CDCl}_{3}\right) \delta 8.99(\mathrm{~d}, J=2.1 \mathrm{~Hz}, 2 \mathrm{H}), 8.67(\mathrm{~m}, J=3.6 \mathrm{~Hz}, 3 \mathrm{H}), 7.75(\mathrm{t}, J=2.8 \mathrm{~Hz}, 1 \mathrm{H})$, $7.49(\mathrm{t}, J=2.6 \mathrm{~Hz}, 1 \mathrm{H}), 7.10(\mathrm{~d}, J=2.4 \mathrm{~Hz}, 1 \mathrm{H}), 4.16(\mathrm{~s}, 3 \mathrm{H}) ;{ }^{13} \mathrm{C} \mathrm{NMR}\left(\mathrm{CDCl}_{3}\right) \delta$ 161.9, 159.6, 134.1, 132.2, 129.3, 126.0, 120.6, 120.1, 105.2, 56.4. IR 1357, 1663, 1703 
$\mathrm{cm}^{-1}$. Anal. Calcd for $\mathrm{C}_{17} \mathrm{H}_{11} \mathrm{~N}_{3} \mathrm{O}_{3}: \mathrm{C}, 66.89 ; \mathrm{H}, 3.61 ; \mathrm{N}, 3.77$. Found: $\mathrm{C}, 67.10 ; \mathrm{H}, 3.42$; N, 3.67 .

4-Methoxy- $N$-(4'-pyrimidinyl)-1,8-naphthalimide (34) mp 266-270 ${ }^{\circ} \mathrm{C} ;{ }^{1} \mathrm{H}$ NMR $\left(\mathrm{CDCl}_{3}\right) \delta 8.69(\mathrm{~m}, J=3.2 \mathrm{~Hz}, 2 \mathrm{H}), 8.56(\mathrm{~s}, 2 \mathrm{H}), 8.43(\mathrm{~d}, J=2.5 \mathrm{~Hz}, 1 \mathrm{H}), 8.22(\mathrm{~d}, J=$ $2.3 \mathrm{~Hz}, 1 \mathrm{H}), 8.12(\mathrm{~d}, J=2.5 \mathrm{~Hz}, 1 \mathrm{H}), 7.92(\mathrm{t}, J=3.0 \mathrm{~Hz}, 1 \mathrm{H}), 6.44$ (d, $J=2.4 \mathrm{~Hz}, 1 \mathrm{H})$, $4.17(\mathrm{~s}, 3 \mathrm{H}) ;{ }^{13} \mathrm{C} \mathrm{NMR}\left(\mathrm{CDCl}_{3}\right) \delta 162.5,160.0,134.9,134.3,133.4,132.1,131.8,130.9$, 128.7, 119.4, 118.4, 105.2, 56.4. IR 1357, $1663,1703 \mathrm{~cm}^{-1}$. Anal. Calcd for $\mathrm{C}_{17} \mathrm{H}_{11} \mathrm{~N}_{3} \mathrm{O}_{3}$ : C, 66.89; H, 3.61; N, 3.77. Found: C, 66.67; H, 3.71; N, 3.68 .

4-Methoxy- $N$-(3'-quinolyl)-1,8-naphthalimide (35) mp 201-205 ${ }^{\circ} \mathrm{C}$; ${ }^{1} \mathrm{H}$ NMR $\left(\mathrm{CDCl}_{3}\right)$ $\delta 8.85(\mathrm{~s}, 1 \mathrm{H}), 8.68(\mathrm{~m}, J=4.9 \mathrm{~Hz}, 3 \mathrm{H}), 8.21(\mathrm{~d}, J=2.4 \mathrm{~Hz}, 2 \mathrm{H}), 7.85(\mathrm{~d}, J=2.8 \mathrm{~Hz}$, 1H), $7.79(\mathrm{~m}, J=4.4 \mathrm{~Hz}, 2 \mathrm{H}), 7.59(\mathrm{t}, J=3.3 \mathrm{~Hz}, 1 \mathrm{H}), 7.12(\mathrm{~d}, J=2.8 \mathrm{~Hz}, 1 \mathrm{H}), 4.21(\mathrm{~s}$, $3 \mathrm{H}) ;{ }^{13} \mathrm{C} \mathrm{NMR}\left(\mathrm{CDCl}_{3}\right) \delta 164.1,161.5,150.5,147.3,135.8,134.1,132.3,130.2,129.8$, 129.5, 129.4, 129.2, 128.2, 128.0, 127.1, 126.2, 123.9, 122.5, 105.5, 56.4. IR 1360, 1661, $1707 \mathrm{~cm}^{-1}$. Anal. Calcd for $\mathrm{C}_{22} \mathrm{H}_{14} \mathrm{~N}_{2} \mathrm{O}_{3}: \mathrm{C}, 74.58 ; \mathrm{H}, 3.95 ; \mathrm{N}, 7.91$. Found: $\mathrm{C}, 74.85 ; \mathrm{H}$, $4.07 ; \mathrm{N}, 8.02$.

4-Methoxy- $N$-(2'-methoxy phenyl)-1,8-naphthalimide (36) mp 205-209 ${ }^{\circ} \mathrm{C} ;{ }^{1} \mathrm{H}$ NMR $\left(\mathrm{CDCl}_{3}\right) \delta 8.62(\mathrm{~m}, J=3.8 \mathrm{~Hz}, 3 \mathrm{H}), 7.73(\mathrm{t}, J=3.3 \mathrm{~Hz}, 1 \mathrm{H}), 7.45(\mathrm{t}, J=3.3 \mathrm{~Hz}, 1 \mathrm{H})$, $7.27(\mathrm{~d}, J=3.0 \mathrm{~Hz}, 1 \mathrm{H}), 7.11(\mathrm{~m}, J=3.8 \mathrm{~Hz}, 3 \mathrm{H}), 4.15(\mathrm{~s}, 3 \mathrm{H}), 3.82(\mathrm{~s}, 3 \mathrm{H}) ;{ }^{13} \mathrm{C} \mathrm{NMR}$ $\left(\mathrm{CDCl}_{3}\right) \delta 163.9,160.9,133.9,132.0,130.2,130.0,128.9,126.0,124.5,123.8,122.8$ 
121.0, 112.0, 105.3, 56.3, 55.8. IR 1370, $1665,1708 \mathrm{~cm}^{-1}$. Anal. Calcd for $\mathrm{C}_{20} \mathrm{H}_{15} \mathrm{NO}_{4}$ : C, 72.07; H, 4.50; N, 4.20. Found: C, 71.84; H, 4.61; N, 4.18.

4-Methoxy- $N$-(3'-methoxy phenyl)-1,8-naphthalimide (37) mp 219-221 ${ }^{\circ} \mathrm{C} ;{ }^{1} \mathrm{H}$ NMR $\left(\mathrm{CDCl}_{3}\right) \delta 8.64(\mathrm{~m}, J=3.8 \mathrm{~Hz}, 3 \mathrm{H}), 7.78(\mathrm{t}, J=3.2 \mathrm{~Hz}, 1 \mathrm{H}), 7.45(\mathrm{t}, J=3.1 \mathrm{~Hz}, 1 \mathrm{H})$, $7.10(\mathrm{~d}, J=2.5 \mathrm{~Hz}, 1 \mathrm{H}), 7.05(\mathrm{~d}, J=2.5 \mathrm{~Hz}, 1 \mathrm{H}), 6.92(\mathrm{~m}, J=4.0 \mathrm{~Hz}, 2 \mathrm{H}), 4.16(\mathrm{~s}, 3 \mathrm{H})$, $3.83(\mathrm{~s}, 3 \mathrm{H}) ;{ }^{13} \mathrm{C} \mathrm{NMR}\left(\mathrm{CDCl}_{3}\right) \delta 164.7,161.1,136.9,134.2,132.0,129.9,129.0,126.1$, 120.8, 115.1, 114.3, 115.2, 56.4, 55.4. IR 1375, 1657, $1707 \mathrm{~cm}^{-1}$. Anal. Calcd for $\mathrm{C}_{20} \mathrm{H}_{15} \mathrm{NO}_{4}: \mathrm{C}, 72.07 ; \mathrm{H}, 4.50 ; \mathrm{N}, 4.20$. Found: C, 71.99; H, 4.61; N, 4.28.

4-Methoxy- $N$-(4'-methoxy phenyl)-1,8-naphthalimide (38) mp 265-268 ${ }^{\circ} \mathrm{C} ;{ }^{1} \mathrm{H}$ NMR (DMSO) $\delta 8.69(\mathrm{~m}, J=3.2 \mathrm{~Hz}, 4 \mathrm{H}), 7.74(\mathrm{t}, J=3.0 \mathrm{~Hz}, 1 \mathrm{H}), 7.24(\mathrm{~d}, J=2.7 \mathrm{~Hz}, 1 \mathrm{H})$, $7.07(\mathrm{~d}, J=2.6 \mathrm{~Hz}, 3 \mathrm{H}), 4.14(\mathrm{~s}, 3 \mathrm{H}), 3.87(\mathrm{~s}, 3 \mathrm{H}) ;{ }^{13} \mathrm{C}$ NMR (DMSO) $\delta 161.1,159.5$, 133.9, 132.0, 129.7, 129.0, 128.3, 126.1, 123.7, 122.7, 115.3, 114.8, 105.4 56.3, 55.6. IR $1352,1669,1701 \mathrm{~cm}^{-1}$. Anal. Calcd for $\mathrm{C}_{20} \mathrm{H}_{15} \mathrm{NO}_{4}: \mathrm{C}, 72.07 ; \mathrm{H}, 4.50 ; \mathrm{N}, 4.20$. Found: C, $71.77 ; \mathrm{H}, 4.33 ; \mathrm{N}, 4.18$.

4-Methoxy- $\mathrm{N}$-(3',5'-dimethoxy phenyl)-1,8-naphthalimide (39) mp $251-253{ }^{\circ} \mathrm{C} ;{ }^{1} \mathrm{H}$ $\operatorname{NMR}\left(\mathrm{CDCl}_{3}\right) \delta 8.69(\mathrm{~m}, J=2.8 \mathrm{~Hz}, 2 \mathrm{H}), 8.45(\mathrm{~d}, J=2.7 \mathrm{~Hz}, 1 \mathrm{H}), 8.11(\mathrm{~d}, J=2.8 \mathrm{~Hz}$, $1 \mathrm{H}), 7.94(\mathrm{~d}, J=3.0 \mathrm{~Hz}, 1 \mathrm{H}), 5.89(\mathrm{~m}, J=3.2 \mathrm{~Hz}, 3 \mathrm{H}), 4.17(\mathrm{~s}, 3 \mathrm{H}), 3.78(\mathrm{~s}, 6 \mathrm{H}) ;{ }^{13} \mathrm{C}$ $\operatorname{NMR}\left(\mathrm{CDCl}_{3}\right) \delta 161.7,160.0,148.3,134.9,134.3,133.4,131.8,131.0,128.7,119.4$ 118.4, 56.3, 55.6. IR 1317, $1664,1707 \mathrm{~cm}^{-1}$. Anal. Calcd for $\mathrm{C}_{21} \mathrm{H}_{17} \mathrm{NO}_{5}$ : C, 69.42; $\mathrm{H}$, 4.68; N, 3.86. Found: C, 69.23; H, 4.71; N, 3.98. 
4-Methoxy-N-(2'-thiazolyl)-1,8-naphthalimide (40) mp 269-273 ${ }^{\circ} \mathrm{C} ;{ }^{1} \mathrm{H}$ NMR $\left(\mathrm{CDCl}_{3}\right)$ $\delta 8.69(\mathrm{~m}, J=2.8 \mathrm{~Hz}, 2 \mathrm{H}), 8.45(\mathrm{~d}, J=2.1 \mathrm{~Hz}, 1 \mathrm{H}), 8.12(\mathrm{~d}, J=2.1 \mathrm{~Hz}, 1 \mathrm{H}), 7.94(\mathrm{t}, J=$ $3.0 \mathrm{~Hz}, 1 \mathrm{H}), 7.07(\mathrm{~d}, J=1.3 \mathrm{~Hz}, 1 \mathrm{H}), 6.53(\mathrm{~d}, J=1.2 \mathrm{~Hz}, 1 \mathrm{H}), 4.15(\mathrm{~s}, 3 \mathrm{H}) ;{ }^{13} \mathrm{C} \mathrm{NMR}$ $\left(\mathrm{CDCl}_{3}\right) \delta 160.0,138.8,134.9,134.3,133.4,132.1,131.8,131.1,128.7,119.4,118.4$ 108.9, 56.4. IR 1342, 1681, $1709 \mathrm{~cm}^{-1}$. Anal. Calcd for $\mathrm{C}_{16} \mathrm{H}_{10} \mathrm{~N}_{2} \mathrm{SO}_{3}$ : C, 61.94; $\mathrm{H}, 3.23$; N, 9.03. Found: C, 62.03; H, 3.31; N, 8.98.

4-Methoxy- $\mathrm{N}$-(6'-quinolyl)-1,8-naphthalimide (41) mp 191-193 ${ }^{\circ} \mathrm{C} ;{ }^{1} \mathrm{H}$ NMR $\left(\mathrm{CDCl}_{3}\right)$ $\delta 8.99(\mathrm{~s}, 1 \mathrm{H}), 8.66(\mathrm{~m}, J=5.1 \mathrm{~Hz}, 3 \mathrm{H}), 8.28(\mathrm{~d}, J=2.5 \mathrm{~Hz}, 1 \mathrm{H}), 8.20(\mathrm{~d}, J=2.5 \mathrm{~Hz}$, 1H), $7.84(\mathrm{~m}, J=6.7 \mathrm{~Hz}, 3 \mathrm{H}), 7.46(\mathrm{t}, J=2.9 \mathrm{~Hz}, 1 \mathrm{H}), 7.41(\mathrm{~d}, J=2.6 \mathrm{~Hz}, 1 \mathrm{H}), 4.16(\mathrm{~s}$, $3 \mathrm{H}) ;{ }^{13} \mathrm{C} \mathrm{NMR}\left(\mathrm{CDCl}_{3}\right) \delta 164.9,164.7,161.4,151.2,147.9,136.4,134.2,132.2,130.9$, $130.3,129.9,129.4,128.6,127.9,126.2,123.8,122.4,121.5,115.0,105.5,56.4$. IR 1376, 1656, $1703 \mathrm{~cm}^{-1}$. Anal. Calcd for $\mathrm{C}_{22} \mathrm{H}_{14} \mathrm{~N}_{2} \mathrm{O}_{3}: \mathrm{C}, 74.58 ; \mathrm{H}, 3.95 ; \mathrm{N}, 7.91$. Found: C, 74.33; H, 3.87; N, 7.83.

4-Methoxy-N-(2'-naphthyl)-1,8-naphthalimide (42) mp 177-179 ${ }^{\circ} \mathrm{C} ;{ }^{1} \mathrm{H}$ NMR $\left(\mathrm{CDCl}_{3}\right)$ $\delta 8.67(\mathrm{~m}, J=4.8 \mathrm{~Hz}, 3 \mathrm{H}), 7.99(\mathrm{~d}, J=2.1 \mathrm{~Hz}, 1 \mathrm{H}), 7.83(\mathrm{~m}, J=6.1 \mathrm{~Hz}, 3 \mathrm{H}), 7.76(\mathrm{t}, J=$ $2.9 \mathrm{~Hz}, 1 \mathrm{H}), 7.55(\mathrm{~m}, J=3.6 \mathrm{~Hz}, 2 \mathrm{H}), 7.42(\mathrm{~d}, J=2.7 \mathrm{~Hz}, 1 \mathrm{H}), 7.11(\mathrm{~d}, J=2.4 \mathrm{~Hz}, 1 \mathrm{H})$; ${ }^{13} \mathrm{C} \mathrm{NMR}\left(\mathrm{CDCl}_{3}\right) \delta 164.2,161.2,134.8,134.6,133.9,133.6,133.0,132.1,130,0,129.3$, $127.9,127.8,126.7,126.3,123.9,122.5,115.3,105.4,56.4$. IR 1358, $1661,1701 \mathrm{~cm}^{-1}$. 
Anal. Calcd for $\mathrm{C}_{23} \mathrm{H}_{15} \mathrm{NO}_{3}$ : C, 78.19; H, 4.25; N, 3.97. Found: C, 78.23; H, 4.31; N, 4.08 . 